\title{
The prevalence of Linguatula serrata nymphs in camels slaughtered in Mashhad slaughterhouse, Northeast, Iran
}

\author{
Saeid R. Nourollahi Fard ${ }^{1}$, Nima Ghalekhani ${ }^{2 *}$, Reza Kheirandish ${ }^{1}$, Saeid Fathi ${ }^{2}$, Ehsan Norouzi Asl ${ }^{2}$ \\ ${ }^{1}$ Department of pathobiology, School of veterinary medicine, Shahid Bahonar University of Kerman, Kerman, Iran \\ ${ }^{2}$ School of veterinary medicine, Shahid Bahonar University of Kerman, Kerman, Iran
}

\section{ARTICLE INFO}

Article history:

Received 2 September 2012

Received in revised form 23 September 2012

Accepted 28 November 2012

Available online 28 November 2012

\section{Keywords:}

Linguatula serrata

Camel

Iran

\section{ABSTRACT}

Objective: To evaluate the prevalence of nymphal stages of L. serrata in mesenteric lymph nodes of camels slaughtered in Mashhad slaughterhouse, Northeast of Iran. Methods: For this purpose, mesenteric lymph nodes of 400 camels of different sex and age were examined. The lymph nodes were examined macroscopically and a digestion method was also applied for investigation of samples which was negative macroscopically. Results:The mesenteric lymph nodes of 73 camels out of $400(18.25 \%)$ were infected by L. serrata nymphs.Conclusions:Prevalence of L. serrata nymphs in males and females and different age was not significantly different $(P>0.05)$, but difference was observed between the prevalence in different seasons $(P<0.05)$. The potential importance of these findings to human health is discussed. This is the first report of infection with L. serrate of camels in camels slaughtered at northeast of Iran.

\section{Introduction}

Linguatula serrata Frohlich, 1789 is a zoonotic parasite causing visceral and nasopharyngeal linguatulosis or Halazoun Syndrome in humans. L. serrata is tongue shaped, lightly convex dorsally and flattened ventrally. Males measure $1.8-2.0 \mathrm{~cm}$, while females measure $8-13 \mathrm{~cm}$ in length. Adults inhabit the canine respiratory system as final hosts. The eggs are expelled from the respiratory passages of the canine and, when swallowed by an intermediate host, the larva reaches the mesenteric lymph nodes, liver, and other organs, in which it develops to the infective nymphal stage. The final host becomes infected by eating the infected viscera of intermediate hosts[1]. Humans may be infected with linguatula either by ingestion of nymphs of $L$. serrata resulting in a condition called nasopharyngeal linguatulosis or Halzoun syndrome or by ingestion of infective eggs which develop in internal organs resulting in visceral linguatulosis[2,3]. Human infection may occur

\footnotetext{
*Corresponding author: Nima Ghalekhani, School of veterinary medicine, Shahid Bahon University of Kerman, Kerman, Iran.

Tel: 00983412474784

Fax: 00983413222047

E-mail address: ghalekhani.n@gmail.com

Fundation Project: Supported by the research council of Shahid-Bahonar University of Kerman (grant number: 1389.4.16).
}

via consumption of raw or undercooked liver and lymph nodes[2,4]. Linguatolosis has been reported from Iran in human[5-7]. Several studies have been conducted on the prevalence rate of L.serrata in dogs[8-12], sheep[3,13-16], goats[17-19], cattle[20] and camels[20-25].

Camel (Camelus dromedarius) is an important multipurpose popular local animal in semiarid areas of Iran, and more than 200000 dromedary camels are living in the arid and semiarid deserts of eastern provinces of Iran. But now camel raisers try to submit the animals basically for the purpose of meat consumption. In some areas of Iran, people are used to consume camel meat which has good quality and economically fair in comparison to beef and sheep[26,27].

Considering importance of this infection for the human health and of course eating raw regions in Iran, few studies were done to know this parasite and the ways it is transmitted.

In many casese, the infected people are recovered very quickly. Because there are no lethal symptoms for this infection, people do not take it seriously, therefore, it is absolutely necessary to pay attention to the prevention of this this disease and also decrease the intermediate host and domestic animals especially in Iran.

The aim of this study was to determine the prevalence rate of nymphal stages of Linguatula serrata in mesenteric 
lymph nodes of camels slaughtered in Mashhad slaughterhouse, Northeast of Iran and to obtain information about the effects of some factors such as season, age and gender on parasitic infections.

\section{Materials and methods}

\subsection{Sampling}

During a 1-year period (April 2009- March 2009), 400 camels, 168 females and 232 males in three age groups $(<5,5-10$ and $>10$ years old $)$ were selected randomly at the Mashhad slaughterhouse, Northeast of Iran.

Their age was determined on the basis of cameleer information.

\subsection{Preparation method}

For this study, mesenteric lymph nodes were examined grossly for pathological alterations and for the presence of nymphal stage of $L$. serrata. Samples were cut into small pieces and immersed in normal saline $(0.9 \% \mathrm{NaCl})$ solution and left for 5-6 h to allow nymphs to come out from tissue. Recovered nymphs were flattened, dehydrated in ascending grades of ethyl alcohol and cleared in creosote before examining under a stereomicroscope. Then the negative samples were digested in $200 \mathrm{ml}$ of digestion solution containing 5 grams of pepsin and $25 \mathrm{ml}$ hydrochloric acid in $1000 \mathrm{ml}$ distilled water, and incubated at $37^{\circ} \mathrm{C}$ for $24 \mathrm{~h}[19]$.

\subsection{Statistical analysis}

The computer software, SPSS Version 9.0 for Windows (SPSS Inc., Chicago, IL, USA) was used for analysis. To compare relative frequency of infection between different groups of lymph nodes Chi-square tests was used. Differences were considered significant when $P<0.05$.

\section{Results}

The prevalence of L. serrata nymphs in mesenteric lymph nodes of 400 camels slaughtered at Mashhad slaughterhouse, Iran in different sex and age groups are summarized in Table 1 and 2. Seventy three out of $400(18.25 \%)$ camels were

Table 1.

The prevalence rate of $L$. serrata nymphs in 400 camels slaughtered at slaughterhouse Mashhad, Iran in different sex and age group

\begin{tabular}{|c|c|c|c|c|c|}
\hline \multirow{2}{*}{ Age groups(years) } & \multicolumn{2}{|r|}{ Male } & \multicolumn{2}{|c|}{ Female } & \multirow{2}{*}{ Total $(\%)$} \\
\hline & No. of camels & No. of infected camels (\%) & No. of camels & No. of infected camels $(\%)$ & \\
\hline$<<$ & 27 & $3(11.11)$ & 16 & $1(6.25)$ & $4(9.30)$ \\
\hline $5-10$ & 59 & $12(20.33)$ & 65 & $9(13.84)$ & $21(16.93)$ \\
\hline$>10$ & 146 & $31(21.23)$ & 87 & $17(19.54)$ & $48(20.60)$ \\
\hline Total & 232 & $46(19.82)$ & 168 & $27(16.07)$ & $73(18.25)$ \\
\hline
\end{tabular}

Table 2.

The prevalence rate of $L$ serrata nymphs in 400 camels slaughtered at slaughterhouse Mashhad, Iran in different season.

\begin{tabular}{|c|c|c|c|c|c|}
\hline \multirow{2}{*}{ Season } & \multicolumn{2}{|r|}{ Male } & \multicolumn{2}{|r|}{ Female } & \multirow{2}{*}{ Total $(\%)$} \\
\hline & No. of camels & No. of infected camels (\%) & No. of camels & No. of infected camels $(\%)$ & \\
\hline Spring & 71 & $10(14.08)$ & 32 & $5(15.62)$ & 15 (14.56) \\
\hline Fall & 66 & $16(24.24)$ & 34 & $11(32.35)$ & $27(27.00)$ \\
\hline Winter & 43 & $7(16.27)$ & 57 & $4(7.01)$ & $11(11.00)$ \\
\hline Total & 232 & $46(19.82)$ & 168 & $27(16.07)$ & $73(18.25)$ \\
\hline
\end{tabular}

infected with nymph stages of $L$. serrata. 46 out of 232 males $(19.82 \%)$ and twenty seven out of 168 females (16.07\%) were found to be positive. Prevalence of L. serrata nymphs in males and females and different age was not significantly different $(P>0.05)$, but difference was observed between the prevalence in different seasons $(P<0.05)$. The maximum and minimum numbers of parasites in MLNs were 36 and 1, respectively.

\section{Discussion}

Linguatulosis poses veterinary and public health importance in the world including Iran. As intermediate host, one-humped or two humped camel, like the other ruminants, may play a vital role in the life cycle of $L$. serrata. As the camels are mostly kept freely in the pastures, they are very susceptible in persistence of infection and its dissemination[28]. The prevalence rate of $L$. serrata in the dog has been reported from Iran, reported a high prevalence (76.5\%) of L. serrata in stray dogs in Shiraz, Iran[12]. Also, a high prevalence rate of infection with $L$. serrata from dogs has been reported (62.2\%) from Shahre-Kord, Iran[11]. Close contact between dogs and the intermediate host plays an important role in transmission of $L$. serrata in this area. Several studies have been conducted to determine the 
prevalence of linguatulosis in ruminants in Iran and other countries. The prevalence rates of $29.9 \%$ in goats in Shiraz, Iran[19], 49.1\% in goats in Kerman, Iran[18], 16.1\% in Sheep in Kerman, Iran[13], 14.8\% in cattle in Babol slaughterhouse, Iran[29], 16.22\% in cattle in Kerman, Iran[20], 19\% in cattle of India[30], have been reported from different countries. In this study, $18.25 \%$ of examined camels had L. serrata nymphs in MLNs. Rajabloo et al reported that in the lymph nodes of 64 camels were diagnosed as infected by L. serrata nymphs[25]. In another study, diagnosed L. serrata infection in $16.2 \%$ of examined camels in Kerman, Iran[24]. reported $L$. serrata nymphs in the left lobe of lung of a Two- Humped male camel in Tabriz, Iran[23].showed that mesenteric lymph nodes of $75 \%$ camels, lungs of $29.7 \%$ and livers of $30.4 \%$ camels were infected with Linguatula serrata nymphs[28]. also reported L. serrata nymphs in 21.0\% MLNs and $4.5 \%$ livers of camels[22].

The infection rates in these results were at variance to those observed in the present study. This probably indicates the geographic and climatic changes which affect the survival of the parasite eggs. Apparently, high prevalence rate of infection in this area are a manifestation of climatic parameters that enhance survival of parasite eggs in vegetables, fruits, and water resources, and possibly, the suitable temperature and humidity play important roles in the epidemiology of this infection. The prevalence rate of $18.25 \%$ infection in lymph nodes should be considered as an important risk factor for human being infection. Larval and nymphal stages of $L$. serrata have been recorded from humans in some countries such as Iran[6,7]. Although, the prevalence of L. serrata in camels of Mashhad is high, it seems that the camels have little role in epidemiology of linguatulosis in comparison with sheep and goats. Prevalence of $L$. serrata nymphs in males and females and different age was not significantly different $(P>0.05)$, the infection rate increased with age but difference was observed between the prevalence in different seasons $(P<0.05)$. The nymphs develop in about 5-6 months, according to the lower prevalence in the younger groups. Human infection is as the result of ingestion of third stage larvae of L.serrata found in raw liver or lymph nodes of sheep, goats, cattle and camels. Ingestion of $L$. serrata nymphs can cause halzoun or marrara syndrome that is often characterized by inflammation of the upper respiratory tract, swelling of the submaxillary and cervical lymph nodes and occasionally abscess formation in the eyes or ears[31-38,9], Sporadic incidence of halzoun in humans has also been reported in Iran[7]. The high prevalence of infection observed in ruminants is of concern owing to the zoonotic nature of the parasite and the risk of infection to humans and other animals. Because of the veterinary and medical importance of linguatulosis, we suggest that further serious investigation be conducted in both carnivores and herbivores.

Because of the veterinary and human medical importance of linguatulosis, further investigations in both domestic and wild herbivores and carnivores together with more detailed studies on the occurrence of this infection in humans are suggested. This study showed a high risk of L. serrata for humans and other animal species. Although linguatulosis is difficult to diagnose, it should not be ignored; adopting proper and reliable diagnostic methods to detect infection in slaughterhouses together with introducing preventive measures are undeniable responsibilities for both veterinary and medical authorities. Adequate cooking of meat and viscera and drinking properly filtered water are crucial in preventing human infections. In addition, this study emphasized the need for a more thorough investigation of the mesenteric lymph nodes during inspection of camels in Iran. Heightened public awareness of the danger of raw food and education of humans on the different aspects of the epidemiology of the parasite together with preventing dogs or other canines ingesting infected material should reduce the risk of infection, and any such measures may also help to diminish other zoonoses.

\section{Conflict of interest statement}

We declare that we have no conflict of interest.

\section{Acknowledgements}

The authors would like to thank the authorities and personnels of Mashhad Slaughterhouse for their cooperation and supports during this study. We are also thankful the authorities of Veterinary School, kerman University for their financial support and cooperation. This study was financially supported by the research council of Shahid-Bahonar University of Kerman (grant number: 1389.4.16).

\section{References}

[1] Rezaei F, Tavassoli M, Javdani M. Prevalence and morphological characterizations of Linguatula serrata nymphs in camels in Isfahan Province, Iran. Veterin Res Forum 2012; 3: 61-65.

[2] Haddadzadeh H, Athari S, Abedini R, Nia P, Nabian S, HajiMohamadi B. One-humped camel (Camelus dromedaries) infestation with Linguatula serrata in Tabriz, Iran. Iran J Arthropod-Born Dis 2010; 4: 54-59.

[3] Rezaei F, Tavassoli M, Mahmoudian A. Prevalence of Linguatula serrata infection among dogs (definitive host) and domestic ruminants (intermediate host) in the North West of Iran. Veterinarni Medicina 2011; 11: 561-567.

[4] Tappe D, Büttner DW. Diagnosis of human visceral pentastomiasis. PLOS Neglected Trop Dis 2009; 3: e320. doi:10.1371/journal.pntd.0000320.

[5] Rokni MB. The present status of human helminthic diseases in 
Iran. Ann Trop Med Parasitol 2008; 102: 283-295.

[6] Anaraki Mohammadi G, Mobedi I, Ariaiepour M, Pourmohammadi Z, Zare Bidaki M. A case report of nasopharyngeal linguatuliasis in Tehran, Iran and characterization of the isolated Linguatula serrata. Iranian J Parasitol 2008; 3: 53-55.

[7] Maleky F. A case report of Linguatula serrata in human throat from Tehran, central Iran. Ind J Med Sci 2001; 55: 439-441.

[8] Dincer S. Prevalence of Linguatula serrata in stray dogs and animals slaughtered at Elazing abattoir (Turkey). Veterinary Faculty Dergisi Ankara University 1982; 29: 324-330.

[9] Yagi H, EL-Bahari S, Mohamed HA, Ahmed ERS, Mustafa B, Mahmoud Saad MB. The Marrara syndrome - a hypersensivity reaction of the upper respiratory tract and buccopharyngeal mucosa to nymphs of Linguatula serrata. Acta Tropica 1996; 62: 127-134.

[10] Rasoli S, Amniattalab A, Sadagian M, Hajikarimlo B, Azizpour Sarijeh A, Jafari K. A servey on the prevalence rate of Linguatula serrata in stray dogs of the city of urmia. Veterin J (Tabriz) 2010; 4: 765-771.

[11] Meshgi B, Asgarian O. Prevalence of Linguatula serrata infection in stray dogs of Shahrekord, Iran. J Vet Med Ser B 2003; 50: 466-467.

[12] Oryan A, Sadjjadi SM, Mehrabani D, Rezaei M. The status of Linguatula serrata infection of stray dogs in Shiraz, Iran. Comp Clin Pathol 2008; 17: 55-60.

[13] Nourollahi Fard SR, Kheirandish R, Asl EN, Fathi S. Mesenteric and mediastinal lymph node infection with Linguatula serrata nymphs in sheep slaughtered in Kerman slaughterhouse, southeast Iran. Trop Anim Health Prod 2011; 43: 1-3.

[14] Yakhchali M, Tehrani AA. Pathological changes in mesenteric lymph nodes infected with $L$. serrata nymphs in Iranian sheep. Revue Méd Vét 2011; 162: 396-399.

[15] Gül A, Değer S, Denizhan V. The prevalence of Linguatula serrata nymphs in sheep in the Van Province. Türkiye Parazitoloji Dergisi 2009; 33: 25-27

[16] Tavassoli M, Tajik H, Dalir-Naghadeh B, Hariri F. Prevalence of Linguatula serrata nymphs and gross changes of infected mesenteric lymph nodes in sheep in Urmia, Iran. Small Rumin Res 2007; 72: 73-76.

[17] Rezaei H, Ashrafihelan J, Nematollahi A, Mostafavi E. The prevalence of Linguatula serrata nymphs in goats slaughtered in Tabriz, Iran. J Parasit Dis 2012; DOI 10.1007/s12639-012-0104-5.

[18] Nourollahi Fard SR, Kheirandish R, Norouzi Asl E, Fathi S. The prevalence of Linguatula serrata nymphs in goats slaughtered in Kerman slaughterhouse, Kerman, Iran. Vet Parasitol 2010; 171: 176-178.

[19] Razavi SM, Shekarforoush SS, Izadi M. Prevalence of Linguatula serrata nymphs in goats in Shiraz, Iran. Small Rumin Res 2004; 54: 213-217.

[20] Nourollahi Fard SR, Kheirandish R, Norouzi Asl E, Fathi S. The Prevalence of Linguatula serrata nymphs in mesenteric lymph nodes in cattle. Amer J Animal Veterin Sci 2010; 5: 155-158.

[21] Oryan A, Khordadmehr M, Ranjbar VR. Prevalence, biology, pathology, and public health importance of linguatulosis of camel in Iran. Trop Anim Health Prod 2011; 43: 1225-1231. DOI 10.1007/ s11250-011-9830-4.

[22] Shakerian A, Shekarforoush SS, Ghafari Rad H. Prevalence of Linguatula serrata nymphs in one humped camel (Camelus dromedarius) in Najaf Abad, Iran. Res Veterin Sci 2008; 84: 243-245.

[23] Haddadzadeh H, Shamsadin Athari S, Hajimohammadi B. The first record of Linguatula serrata infection of two-humped camel (Camelus bactrinus) In Iran. Iran J Parasitol 2009; 4: 59-61.

[24] Radfar MH, Fathi S, Asgarinezhad H, Norouzi Asl E. Prevalence of Linguatula serrata nymphs in one humped camel (Camelus dromedarius) in Southeast of Iran. Sci Parasitol 2010; 11: 199-202.

[25] Rajabloo M, Youssefi MR, Majidirad M, Kordafshari S, Fallahomrani V, Nazaralipour G, et al. Prevalence of Linguatula serrata nymphs in one humped camel (Camelus dromedarius) in Tehran, Iran. Global Veterinaria 2011; 6: 438-440.

[26] Mowlavi G, Massoud J, Mobedi I. Hydatidosis and testicular filariasis (D. evansi) in camel (C. dromedaries) in central part of Iran. Iranian J Publ Health 1997; 25: 21-28.

[27] Rahbari S, Bazargani TT. Blood parasites in camels of Iran. J Vet Parasitol 1995; 9: 45-46.

[28] Tajik H, Tavassoli M, Khani H, Javadi S. Prevalence of Linguatula serrata nymphs in slaughtered camels of Iran. J Camel Prac Res 2007; 14: 69-71.

[29] Youssefi MR, Hadizadeh Moalem SH. Prevalence of Linguatula serrata nymphs in cattle in Babol Slaughterhouse, North of Iran. World J Zool 2010; 5: 197-199.

[30] Ravindran R, Lakshmanan B, Ravishankar C, Subramanian H. Prevalence of Linguatula serrata in domestic ruminants in south India. Southeast Asian J Trop Med Public Health 2008; 39: 5.

[31] Yilmaz H, Cengiz ZT, Cicek M, Dulger AC. A nasopharyngeal human infestation caused by Linguatula serrata nymphs in Van Province: a case report. Turkiye Parazitoloji Dergisi 2011; 35: 47-49.

[32] Koehsler M, Walochnik J, Georgopoulos M, Pruente C, Boeckeler W, Auer H, et al. Linguatula serrata tongue worm in human eye, Austria. Emerg Infect Dis 2011; 17: DOI: 10.3201/eid1705.100790.

[33] Yao MH, Wu F, Tang LF. Human pentastomiasis in China: case report and literature review. J Parasitol 2008; 94: 1295-1298.

[34] Lai C, Wang XQ, Lin L, Gao DC, Zhang HX, Zhang YY, et al. Imaging features of pediatric pentastomiasis infection: a case report. Korean J Radiol 2010; 11: 480-484.

[35] Siavoshi MR, Asmar M, Vatankhah A. Nasopharyngeal pentastomiasis (halzoun): report of 3 cases. Iran J Med Sci 2002; 27: 191-192.

[36] TabaTabaVakili S, Abbasi A, Mobedi I, Movafaghi S, Fereidooni F, Ebrahimi Daryani N. Report of a case with small bowel obstruction by a rare parasite (Pentastomiasis). Govaresh 2012; 17: $55-59$.

[37] Thamprasert MD. Visceral pentastomiasis: A case report. Chiang Mai Mad Bull 1922; 31: 135-138.

[38] Ogun Y. Pentasomide infestation. Ann Afr Med 2011; 10: 62-63. 